\title{
Reducing Phase Noise in Multi-phase Oscillators
}

\author{
Paolo Maffezzoni, Senior Member, IEEE, Bichoy Bahr, Student Member, IEEE, Zheng Zhang, Student Member, \\ IEEE, and Luca Daniel, Member, IEEE
}

\begin{abstract}
This paper investigates phase noise mechanism in arrays of resonant LC oscillators. Such arrays represent today a promising solution for the generation of multi-phase signals needed in several advanced applications. The analysis presented in this paper relies on consolidated phase-domain macromodels as well as on the original concept of noise transfer function illustrated herein. The proposed analysis sheds new light on noise generation in oscillator arrays and is able to explain certain noise degradation effects observed in nonreciprocal coupling networks. Phase-domain simulation together with noise transfer function concept provide a very efficient computational tool for rapid calculations of phase response and output noise. Thanks to this efficient tool and to the gained qualitative understanding, we are able to propose a chain array configuration enhanced by the injection of a clean, low-noise, signal. In this paper, it is shown how the injected chain array can provide the prescribed phase separation while significantly reducing output phase noise.
\end{abstract}

Index Terms-Multi-phase oscillators, noise reduction, noise transfer function.

\section{INTRODUCTION}

Arrays of weakly coupled resonant oscillators can be employed to generate multi-phase harmonic signals, i.e. sinusoids with the same oscillating frequency and with prescribed phase separations. Multi-phase signals are now indispensable in many advanced emerging applications, such as in extremelyhigh frequency synthesis and multiphase clock distribution [1]-[3], as well as in brain-inspired parallel computing for data analysis [4]. For such applications, stringent phase-noise specifications are required.

Previous studies and experimental evidences have shown how coupled oscillators can exhibit a reduction of their phase noise compared to the free-running case [5], [6]. More specifically, the phase noise spectrum near the carrier is reduced by a factor proportional to the number $N$ of stages in the array, while the noise spectrum far from the carrier remains almost unchanged. Such a noise reduction effect is commonly not enough to meet noise specifications. In fact, the noise spectrum near the carrier continues to be shaped as $1 / f^{2}$ (or $1 / f^{3}$ down to the corner frequency where flicker noise gets dominant) even if reduced compared to the free-running case. In addition, it has been shown that certain array configurations, such as nonreciprocal unilaterally coupled chain arrays, may result in unexpected deterioration of the noise spectrum with the ap-

P. Maffezzoni is with the Politecnico di Milano, Milan, Italy. E-mail: pmaffezz@elet.polimi.it.

B. Bahr, Z. Zhang and L. Daniel are with the Massachusetts Institute of Technology (MIT), Cambridge, MA, USA. E-mail: (bichoy,z_zhang,luca)@mit.edu.

Copyright (c) 2016 IEEE. Personal use of this material is permitted. However, permission to use this material for any other purposes must be obtained from the IEEE by sending an email to pubs-permissions@ieee.org. pearance of spurious peaks [5], [6]. This anomalous behavior and performance limitations call for further investigations.

In this paper, we use the phase-domain macromodel presented in [6] and the concept of Noise Transfer Function (NTF) to shed new light on phase-noise mechanism in oscillator arrays. Thanks to this understanding, we demonstrate that the noise performance of the multi-phase chain array can be definitely enhanced, even at low frequencies, by properly injecting an external clean low-noise signal. Such a clean signal is in fact available in the majority of frequency synthesizers and clock generation systems and is obtained by locking one oscillator (within a PLL or with a pulsed injection) to a stable low-frequency reference (i.e., the output of a crystal oscillator). The problem with an external injection is that it may disrupt the correct phase separation if not properly dimensioned. In this paper, we show how injection strength can be set so as to reduce noise while preserving the prescribed phase separation.

The novel contributions of this paper may be summarized as follows:

1) We investigate the form of the NTFs that describe how the noise sources internal to oscillators are transferred to the output phase noise. We derive how such NTFs depend on the specific choices of array topologies.

2) A behavioral method is provided that allows modeling internal noise sources in locked oscillators through a single macro noise source.

3) A chain array topology enhanced by the injection of a clean, low-noise, signal is presented. It is shown that the proposed array provides multi-phase signals with $\pi / N$ phase separation while improving noise spectrum over a wide frequency band. Results are checked, in a few test cases, via comparisons with SpectreRF simulations.

The topics listed above are organized in the paper as follows: Sec. II describes oscillator array structure and review its phase-domain model. In Sec. III, we illustrate phase noise modeling for individual free-running or locked oscillators and then we describe array phase noise analysis and NTF concept. In Sec. IV, we focus on the relevant case of a chain array and derive the conditions under which an external injection becomes effective in noise reduction. Finally, Sec. V is devoted to numerical experiments and validation.

\section{Oscillator ARray}

We consider an array composed with $N$ identical LC resonant CMOS oscillators, as shown in Fig. 1(Top). Each oscillator, when free-running, oscillates autonomously at the angular frequency $\omega_{0}$ and its output voltage, measured at the LC tank nodes, is purely harmonic

$$
V_{0}(t)=V_{M} \cos \left(\omega_{0} t\right),
$$


with $V_{M}$ being the voltage amplitude. To construct the array, oscillators are coupled by a transconductance, implemented by differential-pair transistors. Fig. 1(Top) shows, the coupling circuit between two oscillator stages of the array with index $k$ and $j$. In this example, a differential-pair circuit reads the voltage $V_{k}(t)$ at the $k$ th-stage output and injects a proportional differential current

$$
I_{j}(t)=g_{j k} V_{k}(t)
$$

into the tank nodes of the $j$ th oscillator. The module of parameter $g_{j k}$ corresponds to the transconductance of the associated differential-pair transistor while its sign refers to the way differential current $I_{j}(t)$ is injected into the nodes $n_{j}^{+}$and $n_{j}^{-}$. In this paper, it is conventionally assumed that a positive $g_{j k}$ corresponds to $I_{j}(t)$ exiting node $n_{j}^{+}$and entering $n_{j}^{-}$, as it is the case shown in Fig. 1(Top). A negative $g_{j k}$, instead, corresponds to $I_{j}(t)$ exiting node $n_{j}^{-}$and entering $n_{j}^{+}$and can be implemented by switching the way differentialpair drains are connected to the injected nodes. In the array in Fig. 1(top) coupling is bilateral since a second differential-pair transistor reads the voltage $V_{j}(t)$ of the $j$ th stage and injects a proportional current $I_{k}(t)=g_{k j} V_{j}(t)$ into the $k$ th stage. The array topology can be schematically represented by the system shown in Fig. 1(Bottom) where couplings are indicated by oriented arches of strength $g_{j k}$. Array topology can be described by the conductance matrix $\mathbf{G}=\left\{g_{j k}\right\} \in \mathbb{R}^{N \times N}$ that collects all coupling strength coefficients $g_{j k}$.

It is known that for resonant oscillators with well matched free-running frequency, weak coupling (e.g. with the transconductance of coupling transistors one order of magnitude smaller than that of oscillator transistors) is enough to keep oscillators synchronized. Under this hypothesis, the array response can be realistically simulated with a phase-domain macromodel [7]-[12]. According to this method, the output voltage of the $k$ th oscillator in the array is written as

$$
V_{k}(t)=V_{0}\left(t+\alpha_{k}(t)\right)=V_{M} \cos \left(\omega_{0} t+\omega_{0} \alpha_{k}(t)\right)
$$

where $\alpha_{k}(t)$ represents the time-dependent time shift of the perturbed response with respect to the free-running one and $\phi_{k}(t)=\omega_{0} \alpha_{k}(t)$ is the associated excess phase variable. Then, the time-shift variable and injected current $I_{k}(t)$ are related by the scalar differential equation [7], [8]

$$
\dot{\alpha}_{k}(t)=\Gamma_{k}\left(t+\alpha_{k}(t)\right) I_{k}(t)
$$

where $\Gamma_{k}(t)$ is the periodic phase-sensitivity function to current injection at the tank. It has been found that for LC resonant oscillators $\Gamma(t)$ is sinusoidal and delayed by a $\pi / 2$ phase angle with respect to the output response [16], i.e.

$$
\Gamma_{k}(t)=\Gamma_{M} \cos \left(\omega_{0} t-\pi / 2\right)
$$

The phase response of the whole chain array is governed by the following set of nonlinear differential-algebraic equations

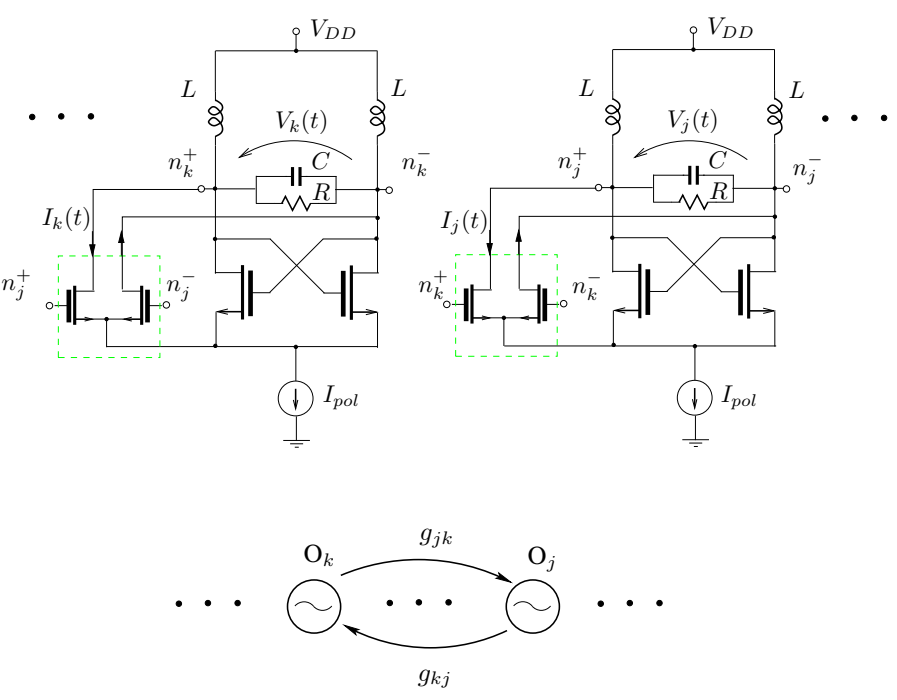

Fig. 1. (Top) Circuit of two of the oscillators forming the array and coupling transistors. (Bottom) Schematic representation.

$$
\begin{array}{r}
\dot{\alpha}_{k}(t)=\Gamma_{k}\left(t+\alpha_{k}(t)\right) I_{k}(t) \\
I_{k}(t)=\sum_{j=1}^{N} g_{k j} V_{j}\left(t+\alpha_{j}(t)\right) .
\end{array}
$$

Array synchronization is achieved if, asymptotically for $t \rightarrow \infty$, the time-shift variables $\alpha_{k}(t)$ approach the waveforms $\widetilde{\alpha}_{k}(t)$ such that the associated phases $\phi_{k}(t)=\omega_{0} \widetilde{\alpha}_{k}(t)$ satisfy

$$
\lim _{t \rightarrow \infty} \phi_{k}(t)-\phi_{j}(t)=\Phi_{k j}
$$

for all $k$ and $j$, where $\Phi_{k j}$ is a constant [13]. In other terms, the phase difference (or phase separation) between any two oscillators converges to a constant value and the oscillator array generates a multi-phase harmonic signal. Numerical integration of (6) allows us to efficiently verify whether synchronization condition (7) is satisfied and to calculate the steady-state phase separations $\Phi_{k j}$ in case of synchronization.

\section{PHASE-NOISE ANALYSIS}

Phase-domain model (3) is suitable to analyze phase-noise effects in oscillator arrays. Noise sources internal to each oscillator produce random fluctuations of the time shift variable $\alpha_{k}(t)$ and of the associated phase $\phi_{k}(t)$ (referred to as phase noise). When many oscillators are coupled to form an array, the internal noise sources of oscillators interact among them through a complex mechanism that results in the final output phase noise. Such a mechanism can be investigated via a two step procedure that consists in modeling noise in the individual uncoupled oscillator through a macro noise source, and then using the phase macromodel (6), augmented with such macro noise sources, to evaluate array phase noise. 


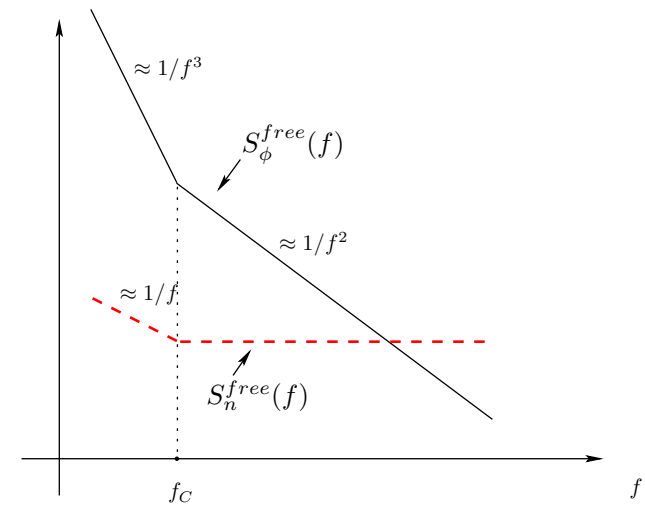

Fig. 2. (Solid line) Output noise spectrum $S_{\phi}^{\text {free }}(f)$ for a free-running oscillator, (Dashed line) spectrum $S_{n}^{f r e e}(f)$ of the associated macro noise source.

\section{A. Individual free-running oscillator}

For a free-running oscillator, phase noise can be described, in a compact way, by the average stochastic equation

$$
\dot{\alpha}(t)=n(t),
$$

where $n(t)$ is a macro noise source, with Power Spectral Density (PSD) $S_{n}(f)$, that accounts for all of the device noise sources internal to the oscillator circuit [17], [18]. The PSD of the phase-noise variable $\phi(t)=\omega_{0} \alpha(t)$ is given by

$$
S_{\phi}(f)=\frac{f_{0}^{2}}{f^{2}} \cdot S_{n}(f)=\operatorname{NTF}(f) \omega_{0}^{2} S_{n}(f),
$$

where $\operatorname{NTF}(f)=1 /|i 2 \pi f|^{2}$ is the noise transfer function (normalized to $\omega_{0}$ ) that describes how the power noise of the source $n(t)$ is transferred to the output, whereas in this paper we denote $i=\sqrt{-1}$ the imaginary unit.

For a given oscillator (i.e. with given circuit parameters) working in free-running mode the output phase-noise spectrum $S_{\phi}^{\text {free }}(f)$ is a known data. In fact, it can be determined either via detailed circuit-level simulations or through laboratory measurements. Such a spectrum is formed of two contributions

$$
S_{\phi}^{f r e e}(f)=\frac{K_{w}}{f^{2}}+\frac{K_{f}}{f^{3}},
$$

due to white and flicker noise sources, respectively, as it is qualitatively portrayed in Fig. 2. In this figure, vertical and horizontal axis are scaled logarithmically and $f$ represents offset frequency. The values of the parameters $K_{w}$ and $K_{f}$ in (10) can be extracted by fitting the shape of the available output spectrum. In view of (9), the PSD of the associated macro noise source $n^{\text {free }}(t)$ is deduced to be

$$
S_{n}^{f r e e}(f)=\frac{K_{w}}{f_{0}^{2}}+\frac{K_{f}}{f_{0}^{2}} \frac{1}{f},
$$

as reported in Fig. 2, where $f_{C}$ represents the corner frequency down which flicker noise becomes relevant.

\section{B. Oscillator locked to a reference}

A clean, low-noise and high-frequency oscillator can be obtained by locking one oscillator to a stable low-frequency

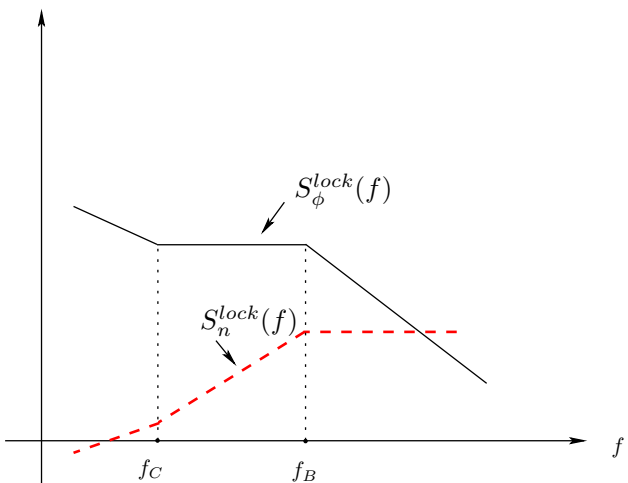

Fig. 3. (Solid line) Output noise spectrum $S_{\phi}^{l o c k}(f)$ of a locked oscillator, (Dashed line) spectrum $S_{n}^{\text {lock }}(f)$ of the associated macro noise source.

reference (i.e. a crystal oscillator) within a control system, such as a Phase-Locked-Loop (PLL) or a Pulsed-InjectionLocked-Oscillator (PILO) [14]. The control system performs a low-pass filtering of the oscillator phase noise. Hence, denoting $f_{B}$ the filter pole frequency (i.e. the control bandwidth), the output spectrum of the locked oscillator results of the type

$$
S_{\phi}^{l o c k}(f)=\left(K_{w}+\frac{K_{f}}{f}\right) \frac{1}{\left|i f+f_{B}\right|^{2}}
$$

as it is qualitatively shown in Fig. 3 under the realistic hypothesis that $f_{B}>>f_{C}$. In view of (9), we derive that the macro noise source $n^{\text {lock }}(t)$ to be associated to a clean low-noise oscillator has the PSD

$$
S_{n}^{l o c k}(f)=\left(K_{w}+\frac{K_{f}}{f}\right) \frac{1}{f_{0}^{2}} \frac{f^{2}}{\left|i f+f_{B}\right|^{2}},
$$

which vanishes for $f<<f_{B}$, as shown in Fig. 3 .

\section{Oscillator array}

The oscillator array is formed by coupling $N$ free-running LC oscillators as shown in Fig. 1. In addition, one lownoise oscillator (whose noise model has been described in subsection III-B) may be present in the system. An example of such an array topology is investigated in the next section and is shown in Fig. 6. The low-noise oscillator is supposed to inject unilaterally into the other stages of the array so that its behavior (and its low noise output) is not affected by array operation.

To study phase noise in the oscillator array, the macro noise source $n_{k}(t)$ associated to the $k$ th oscillator is added to equation (6a), i.e.

$$
\dot{\alpha}_{k}(t)=\Gamma_{k}\left(t+\alpha_{k}(t)\right) I_{k}(t)+n_{k}(t) .
$$

For oscillators that were running in free mode before being coupled, the noise source $n_{k}(t)$ in (14) has PSD of the type (11), whereas for the low-noise oscillator, the associated noise source $n_{k}(t)$ has PSD of the type (13).

Combining (14) with (6b), we are led to the following set of stochastic differential equations

$$
\dot{\alpha}_{k}(t)=\Gamma_{k}\left(t+\alpha_{k}(t)\right) \cdot \sum_{j=1}^{N} g_{k j} V_{j}\left(t+\alpha_{j}(t)\right)+n_{k}(t) \text {. }
$$


The presence of noise sources induces extra random fluctuations $\tau_{k}(t)$ of variables $\alpha_{k}(t)$ around their noiseless regime values $\widetilde{\alpha}_{k}(t)$, i.e.

$$
\alpha_{k}(t)=\widetilde{\alpha}_{k}(t)+\tau_{k}(t) .
$$

Exploiting the fact that $\tau_{k}(t)<<\widetilde{\alpha}_{k}(t)$, in the Appendix it is shown that the PSD of the noise-induced excess phase $\theta_{k}(t)=\omega_{0} \tau_{k}(t)$ for the $k$ th oscillator in the array is given by

$$
S_{\theta_{k}}(f)=\sum_{j=1}^{N} \mathrm{NTF}_{k j}(f) \omega_{0}^{2} S_{n_{j}}(f),
$$

where

$$
\mathrm{NTF}_{k j}(f)=\left|t_{k j}(f)\right|^{2} .
$$

In the expression above, the complex coefficient $t_{k j}(f)$ represents the signal transfer function from input $n_{j}(t)$ of $j$ th oscillator to output phase $\theta_{k}(t)$ of $k$ th oscillator, while $\mathrm{NTF}_{k j}(f)$ is the associated noise transfer function in terms of noise power.

Furthermore, transfer functions $t_{k j}(f)$ are the entries of the complex matrix

$$
\mathbf{T}(f)=\left\{t_{k j}\right\}=\left(i 2 \pi f \mathbf{I}_{N}-\mathbf{A}\right)^{-1},
$$

where $\mathbf{I}_{N}$ is the identity matrix of size $N$, " -1 " denotes inverse operator, while $\mathbf{A}=\left\{a_{k j}\right\} \in \mathbb{R}^{N \times N}$ is defined as follows

$$
\begin{aligned}
& a_{k k}=B \sum_{j=1, j \neq k}^{N} g_{k j} \cos \left(\Phi_{k j}\right) \\
& a_{k j}=-B g_{k j} \cos \left(\Phi_{k j}\right) \quad \text { for } k \neq j,
\end{aligned}
$$

with $B=\omega_{0} \Gamma_{M} V_{M} / 2$, as derived in the Appendix.

In the remainder of this section, we better investigate the form of the signal transfer functions $t_{k j}(f)$. As underlined in the Appendix, for any phase separation $\Phi_{k j}$ that corresponds to a stable solution of (6), the eigenvalues $\lambda_{k}$ of $\mathbf{A}$ are such that: $\lambda_{1}=0$, while remaining ones have negative real part $\Re\left(\lambda_{k}\right)<0$ for $k=2, \ldots, N$. For exposition simplicity, in what follows we suppose that such eigenvalues are all distinct (this is in fact the case for the chain array topologies that we will consider later). Under this hypothesis, matrix $\mathbf{A}$ has eigenvalue decomposition as follows:

$$
\mathbf{A}=\mathbf{V} \cdot \mathbf{D}_{\lambda} \cdot \mathbf{W}^{T}
$$

where the diagonal matrix $\mathbf{D}_{\lambda}$ collects the $\lambda_{k}$ eigenvalues, whereas the columns of matrix $\mathbf{V}$ are the related eigenvectors $\vec{V}_{k}$. In particular, eigenvector $\vec{V}_{1}$ associated to $\lambda_{1}=0$ spans the null space of matrix A, i.e. A $\cdot \vec{V}_{1}=\overrightarrow{0}$. The columns $\vec{W}_{j}$ of matrix $\mathbf{W}$ defined as $\mathbf{W}^{T}=\mathbf{V}^{-1}$ are the rows of the inverse matrix matrix $\mathbf{V}^{-1}$. From (19), it results

$$
\mathbf{T}(f)=\mathbf{V} \cdot \mathbf{D}_{i n} \cdot \mathbf{W}^{T},
$$

where the diagonal matrix $\mathbf{D}_{i n}$ collects the elements $\left(i 2 \pi f-\lambda_{k}\right)^{-1}$. Expression (22) can be further expanded into

$$
\mathbf{T}(f)=\vec{V}_{1} \vec{W}_{1}^{T} \frac{1}{i 2 \pi f}+\sum_{k=2}^{N} \vec{V}_{k} \vec{W}_{k}^{T} \frac{1}{i 2 \pi f-\lambda_{k}} .
$$

The following qualitative observations are in order.

1) Coupling oscillators to form an array results in filtering the macro noise source $n_{k}(t)$ through transfer functions $t_{k j}(f)$ whose poles are the eigenvalues $\lambda_{k}$ of matrix $\mathbf{A}$.

2) If eigenvalues $\lambda_{k}$ are complex conjugate (i.e. they have a nonzero imaginary part), these transfer functions may give resonance effects with unwanted spikes in the output phase noise spectrum. This phenomenon, which was observed in previous studies [5] and [6], becomes more pronounced when the number $N$ of stages is increased. Such spikes in the output spectrum are removed if the conductance matrix $\mathbf{G}=g_{j k}$ is made symmetric, i.e. if coupling is bilateral with $g_{k j}=g_{j k}$. In this case in fact $\mathbf{A}$ is symmetric and thus its eigenvalues $\lambda_{k}$ are purely real, which eliminates any resonance effect. For these reasons, from now on our analysis will be focused on symmetric arrays.

3) The first term in expansion (23) is the most critical one since its transfer function $\propto 1 / f$, which corresponds to integrating noise in time, produces large phase noise components at low frequencies.

4) Nonzero elements of vector $\vec{W}_{1}$ tell us which noise sources in the array are actually integrated through $1 / f$ transfer function, in other words the noise source $n_{p}(t)$ associated to $p$ th oscillator is integrated in time if and only if the $p$ th element in vector $\vec{W}_{1}$ is non zero. As a result, in order to minimize output noise, vector $\vec{W}_{1}$ should have non zero elements only in correspondence to low-noise oscillators.

We conclude this section by observing that vector $\vec{W}_{1}$ is the eigenvector of $\mathbf{A}^{T}$ associated to $\lambda_{1}=0$ and thus it spans the null space of matrix $\mathbf{A}^{T}$. This is easily seen by transposing (21) and observing that $\mathbf{W}^{T}=\mathbf{V}^{-1}$, which yields

$$
\mathbf{A}^{T}=\mathbf{W} \cdot \mathbf{D}_{\lambda} \cdot \mathbf{W}^{-1} \text {. }
$$

\section{CHAIN ARRAYS WITH CLEAN SIGNAL INJECTION}

An array topology of particular importance is the chain array where only nearest neighbor oscillators are coupled, i.e. $g_{k j} \neq 0$ only for $j \in(k-1, k+1)$. Chain arrays are interesting for implementation reasons since they require a limited number of coupling stages. More importantly, it has been proved that for a synchronized chain array only one steady-state phase separation $\Phi_{k j}$ corresponds to a stable solution of (6) and thus it is observable in practice [6], [15]. This stable phase separation only depends on array topology and coupling strengths, i.e. on conductance matrix $\mathbf{G}$, while it does not depend on initial phase conditions.

In this paper, in particular, we will focus on the chain array topology portrayed in Fig. 4 where neighboring oscillators are connected by a symmetric bilateral coupling of strength $-g$ (with $g$ being the differential-pair transistor transconductance). The chain array is closed at the ends with the first and last stages that are bilaterally coupled with strength $g$. The associated conductance matrix $\mathbf{G}$ is reported in Fig. 5(Top).

For this chain array, it has been proved that when synchronization is achieved, steady-state phase variables are such that:

$$
\Phi_{k+1, k}=\phi_{k+1}(t)-\phi_{k}(t)= \pm \pi / N .
$$




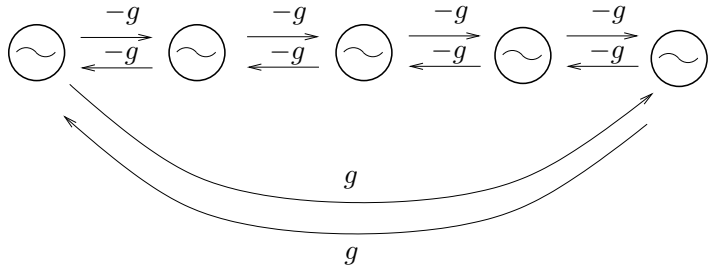

Fig. 4. Chain array topology.

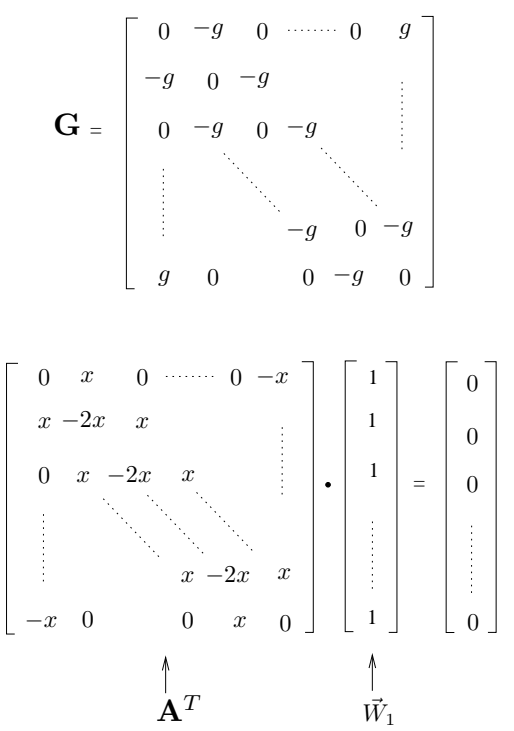

Fig. 5. (Top) Conductance matrix $\mathbf{G}$ for the array in Fig. 4. (Bottom) Matrix $\mathbf{A}^{T}$ and its null space $\vec{W}_{1}$

Thus, denoting $x=g B \cos \left(\Phi_{k+1, k}\right)$, the symmetric matrix $\mathbf{A}=\mathbf{A}^{T}$ exhibits the structure shown in Fig. 5(Bottom) and its null space is spanned by the vector $\vec{W}_{1}$ formed by all ones. This implies that all of the noise sources $n_{k}(t)$ in the array are transferred to the outputs through the critical network function $1 / i 2 \pi f$. We thus expect that for the chain array in Fig. 4 the output power spectra, even if reduced compared to the freerunning case, will continue to be shaped as $1 / f^{2}$ (and $1 / f^{3}$ at the very low frequencies down to $f_{C}$ ).

To improve the noise performance, in what follows we investigate the enhanced array arrangement shown in Fig. 6: in this arrangement the oscillators in the chain array are injected unilaterally with the clean signal provided by a lownoise oscillator labelled $\mathrm{O}_{1}$. The macro noise source $n_{1}(t)$ associated to $\mathrm{O}_{1}$ has the PSD described in (11) which vanishes for $f<f_{B}$. Signal injection from $\mathrm{O}_{1}$ should reduce the smallsignal phase fluctuations induced by noise while minimally affecting the large-signal phases $\phi_{k}(t)$ and related phase separations. To achieve this goal, the injection strength is fixed to a value $g_{r}<<g$. The conductance matrix $\mathbf{G}$ for the injected chain is augmented by an all-zero row (oscillator 1 is not injected by others) and one column collecting injection strength coefficients $g_{r}$ as shown in Fig. 7(Top). Supposing that (25) remains unchanged, and adopting the notations $x=g B \cos \left(\Phi_{k+1, k}\right), x_{r}=g_{r} B \cos \left(\Phi_{k+1, k}\right)$, and $y=-2 x+x_{r}$, the structure of the $\mathbf{A}^{T}$ matrix (i.e. its non zero

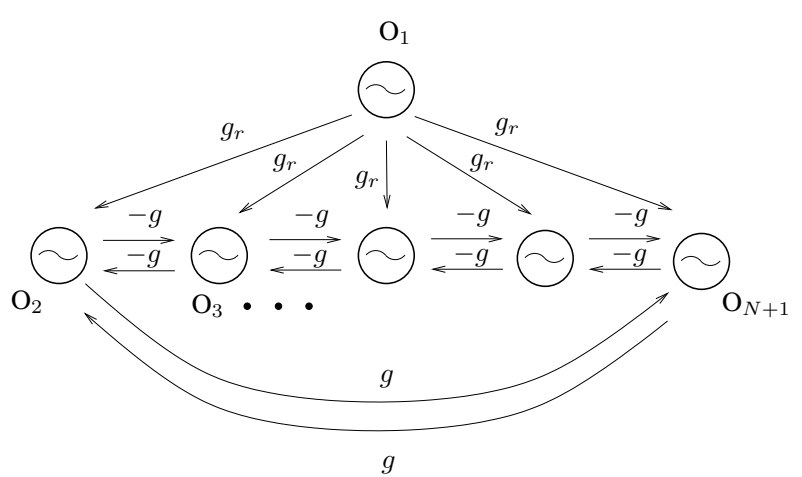

Fig. 6. Chain array injected by the low-noise oscillator $\mathrm{O}_{1}$.
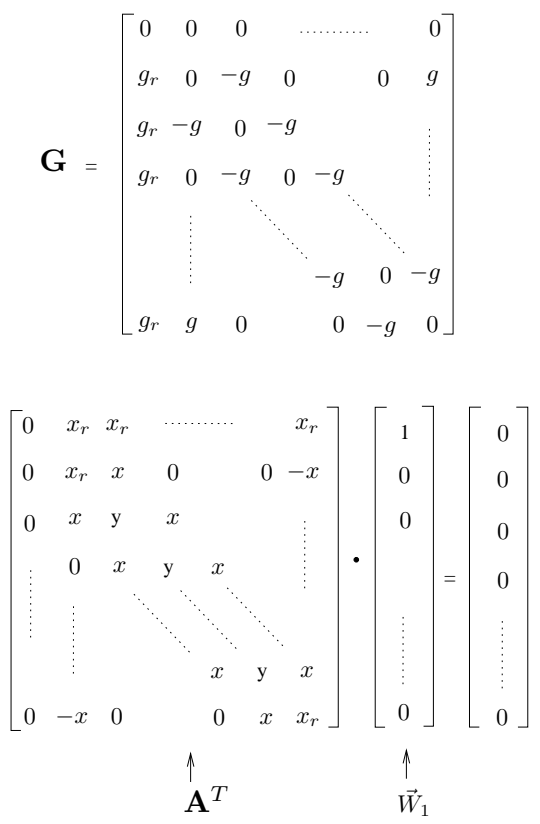

Fig. 7. (Top) Conductance matrix $\mathbf{G}$ for the array with injection in Fig. 6. (Bottom) Matrix $\mathbf{A}^{T}$ and its null space $\vec{W}_{1}$.

entries) is shown in Fig. 7(Bottom). The null-space spanning vector $\vec{W}_{1}$ has a one in the first position and all zeros in the others. This means that in the injected array the only noise source which is transferred through the critical network function $1 / i 2 \pi f$ is that of the clean source $n_{1}(t)$ associated to the locked oscillator. This is expected to improve significantly the array noise performance at the low frequencies.

\section{NumericAl Results}

In this section, we present numerical results for chain arrays formed with $N=5$ identical LC oscillators and for configurations without external injection and with injection. The circuit of each LC oscillator is shown in Fig. 1 with the device parameters reported in table I. A single oscillator is first simulated with the periodic steady state (pss) analysis of SpectreRF and then the $\Gamma(t)$ function is extracted with the method described in [16]. The circuit oscillates at $f_{0}=$ $1.0261 \mathrm{GHz}$ and its output voltage $V_{0}(t)$ and $\Gamma(t)$ function are harmonic as in (1) and (5) with peak values $V_{M}=3.45 \mathrm{~V}$, $\Gamma_{M}=151.8 \mathrm{~A}^{-1}$, respectively. 
TABLE I

PARAMETERS OF THE LC OSCILLATOR

\begin{tabular}{||c|l||}
\hline \hline Parameter & Value \\
\hline \hline$V_{D D}$ & $2.5 \mathrm{~V}$ \\
\hline$I_{p}$ & $640 \mu \mathrm{A}$ \\
\hline$C$ & $0.3 \mathrm{pF}$ \\
\hline$L$ & $40 \mathrm{nH}$ \\
\hline$R$ & $11 \mathrm{k} \Omega$ \\
\hline$(W / L)$ & 30 \\
\hline
\end{tabular}

Oscillator stages are coupled as in Fig. 4, to form a chain array with no injection. A fixed differential-pair transconductance parameter $g=g^{0}=10^{-5} \Omega^{-1}$ is chosen. The phase response of the chain array is then simulated with the model (6), starting from initial random phase values. Fig. 8 shows the time evolution of the $\phi_{k}(t)$ phase variables: the phase difference among nearby oscillators converges to the constant value $\Delta \phi=\pi / 5 \approx 0.628 \mathrm{rad}$ meaning that the oscillator array is synchronized.

Hence, the array with unilateral external injection as described in Fig. 6 is considered: the low-noise oscillator $\mathrm{O}_{1}$ injects unilaterally in the chain stages, numbered from 2 to $N+$ $1=6$, with transconductance strength $g_{r}=g_{r}^{0}=10^{-6} \Omega^{-1}$. Fig. 9 shows the simulated time evolution of the $\phi_{k}(t)$ phase variables for the array with injection: compared to the case with no injection, array synchronization takes a longer time but eventually phase separations among chain array stages, i.e. for $k=2, \ldots, N+1$, converge to $\Delta \phi \approx \pi / 5$ with a relative error which is smaller than $2 \%$. Besides that, chain array oscillators synchronize with the external signal in the way that oscillator $\mathrm{O}_{4}$, in the center of the chain array, is almost in antiphase with $\mathrm{O}_{1}$, i.e. $\phi_{1}(t)-\phi_{4}(t) \approx \pi$. This behavior is fully confirmed by detailed circuit-level simulations with SpectreRF. Fig. 10 reports the oscillators output voltages derived with the phasedomain simulation and model (3) and those obtained through simulation with SpectreRF: the waveforms (after being properly delayed) match with great accuracy. We thus conclude that, for the selected coupling strengths, the prescribed phase separation is preserved in the presence of external injection.

We pass now to analyze noise. To this aim, the output phase noise of the individual free-running oscillator is computed with the pnoise analysis of SpectreRF [19]. For the considered oscillator device, phase noise is dominated by the thermal white noise down to a corner frequency $f_{C}$ of some hundreds hertz. Over the frequency range of interest, the spectrum of the free-running oscillator is well approximated by

$$
S_{\phi}(f) \approx \frac{100}{f^{2}} \operatorname{rad}^{2} / \mathrm{Hz}
$$

which corresponds to (10) with $K_{f}=0$. In view of (9) or (11), the associated macro noise source $n(t)$ has a constant PSD $S_{n}(f)=\widetilde{S}_{n}=10^{-16} \mathrm{rad}^{2} / \mathrm{Hz}$. Noise in each oscillator in the chain array is thus modelled with a macro noise source $n_{j}(t)$ having constant PSD $S_{n_{j}}(f)=\widetilde{S}_{n}$.

For the array configuration with external injection, oscillator $\mathrm{O}_{1}$ is a locked low-noise oscillator whose PSD of the type (12)

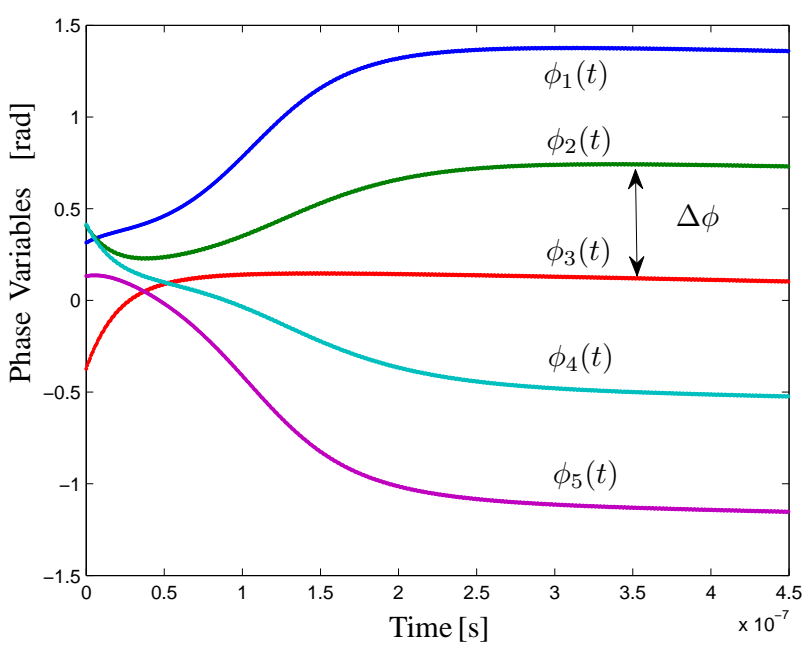

Fig. 8. Phase response of the array with no injection.

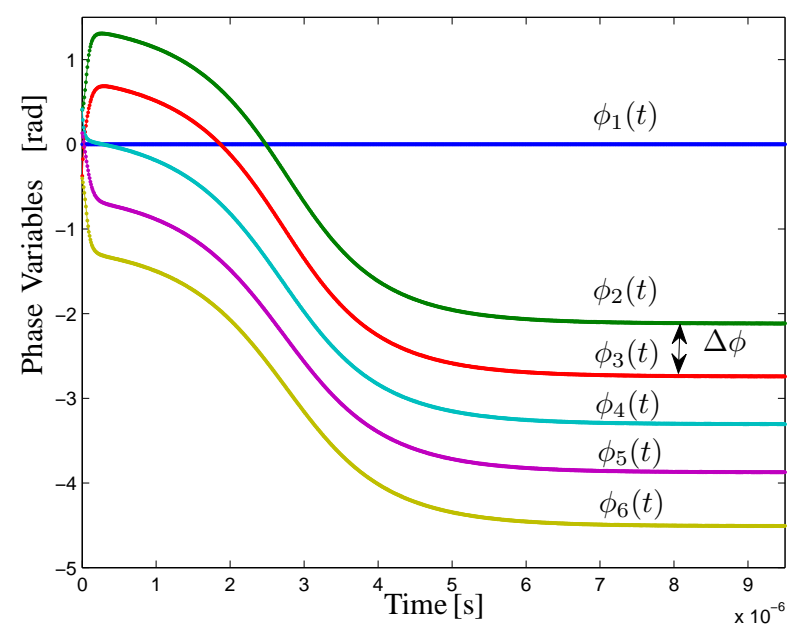

Fig. 9. Phase response of the array with external injection.

is well approximated by

$$
S_{\phi_{1}}(f)=\frac{100}{\left|i f+f_{B}\right|^{2}},
$$

with control bandwidth $f_{B}=10 \mathrm{MHz}$. From (11), we deduce that macro noise source $n_{1}(t)$ has PSD

$$
S_{n_{1}}(f)=\frac{100}{f_{0}^{2}} \frac{f^{2}}{\left|i f+f_{B}\right|^{2}} .
$$

First, we focus on the array with no injection and, starting from the simulated phase separations $\Phi_{k j}$ shown in Fig. 8 and using (19) and (20), we calculate the NTFs. Fig. 11 shows $\mathrm{NTF}_{22}(f)$ describing self-noise transfer from source $n_{2}(t)$ to output $\theta_{2}(t)$ for oscillator number 2 (the same curves are found for the other oscillators in the chain). It also shows $\mathrm{NTF}_{2 j}(f)$, with $j \in(1,3,4,5)$, describing noise transfer from other oscillators. We conclude that, even though such NTFs are attenuated compared to $\operatorname{NTF}(f)=1 /|i 2 \pi f|^{2}$ for 


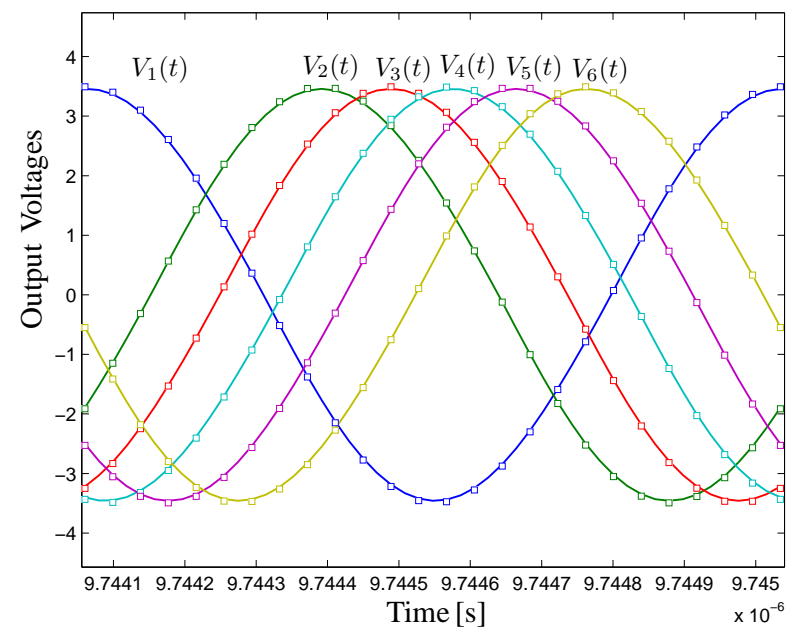

Fig. 10. Output voltages for the array with injection: (Solid line) waveforms obtained with the phase-domain model, (Square marker) waveforms simulated with SpectreRF

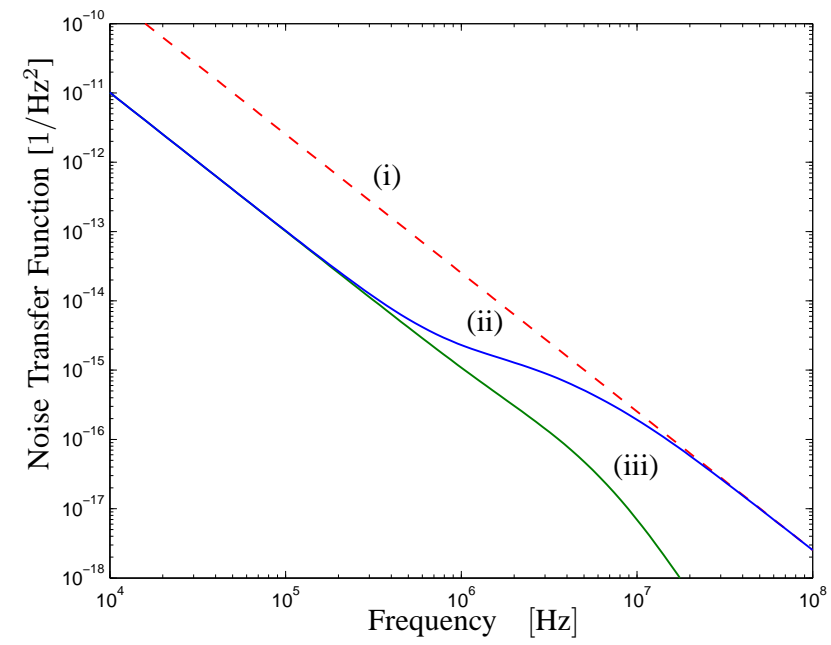

Fig. 11. (i) $\mathrm{NTF}(f)$ for the free-running oscillator, (ii) $\mathrm{NTF}_{22}(f)$ in the array with no injection, (iii) $\mathrm{NTF}_{2 j}(f)$ with $j \in(1,3,4,5)$ in the array with no injection.

the free-running oscillator, they still vary as $1 / f^{2}$ at the low frequencies.

Second, we calculate the NTFs for the array with injection using the simulated phase separations $\Phi_{k j}$ shown in Fig. 9. In this case, we see from Fig. 12 how self-noise function $\mathrm{NTF}_{22}(f)$ and transfer functions $\mathrm{NTF}_{2 j}(f)$ for $j \in(3,4,5,6)$ are significantly reduced down to $\approx 1 \mathrm{MHz}$ where they tend to constant values. The only NTF that keeps varying as $1 / f^{2}$ is the $\mathrm{NTF}_{21}(f)$ that weights clean noise source $n_{1}(t)$. This should reduce the total output phase noise. Fig. 13 shows the total output phase noise $S_{\theta_{2}}(f)$ computed with the phasedomain model for the cases of array without injection and with injection. External injection is seen to yield a remarkable noise reduction at the low frequencies. This result is fully confirmed by circuit-level phase noise simulations with

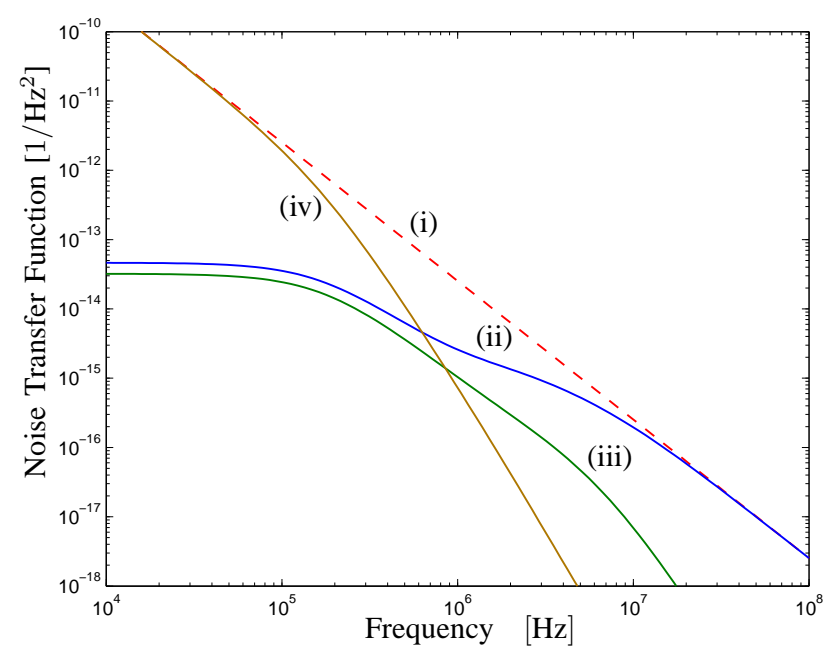

Fig. 12. (i) $\mathrm{NTF}(f)$ for the free-running oscillator, (ii) $\mathrm{NTF}_{22}(f)$ in the array with injection, (iii) $\mathrm{NTF}_{2 j}(f)$ with $j \in(3,4,5,6)$ in the array with injection, (iv) $\mathrm{NTF}_{21}(f)$

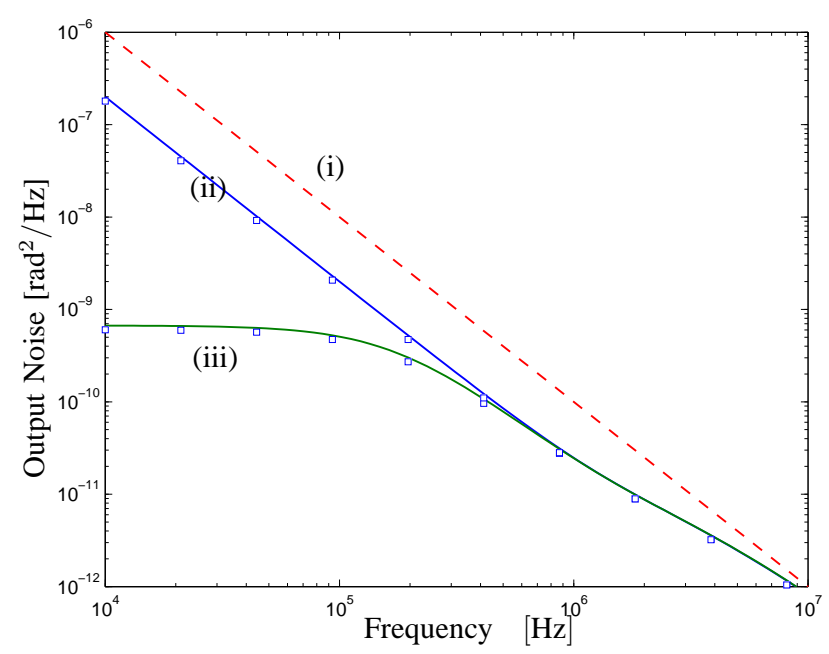

Fig. 13. (i) Phase-noise $S_{\phi}(f)$ in the free-running oscillator. (ii) Output phase-noise $S_{\theta_{2}}(f)$ in the array without injection. (iii) Output phase-noise $S_{\theta_{2}}(f)$ in the array with injection. Solid lines refer to phase-domain simulations, square markers refer to simulations with SpectreRF.

SpectreRF as reported in Fig. 13 for comparison. Detailed SpectreRF simulation is indeed time consuming, (e.g. for the relatively simple case of $N=5$ with external injection, the single simulation requires about 20 minutes on a quad core) and thus it is used only for verification purpose. By contrast, phase domain analysis requires only a few seconds and thus it allows extensive exploration of array performances as a function of parameters values. In the remainder of this section, we exploit the efficiency of the phase domain model to investigate two issues that are relevant for practical implementations. A first issue is connected to the variability of coupling coefficients due to fabrication uncertainty. To study this effect, we assume that coupling transconductance $g$ and injection strength $g_{r}$ undergo random variations around their nominal 
values $g^{0}$ and $g_{r}^{0}$, according to $g=g^{0} \cdot[1+\mathcal{U}(-a, a)]$ and $g_{r}=g_{r}^{0} \cdot[1+\mathcal{U}(-a, a)]$, respectively. The symbol $\mathcal{U}(-a, a)$ denotes a stochastic variable uniformly distributed over the interval $(-a, a)$. Hence, we perform Monte Carlo simulations where for each randomly generated $g$ and $g_{r}$ values, we simulated the phase-domain response of the injected array and determine the steady-state phase-difference values. Fig. 14 shows the statistical distribution of the asymptotic phase difference $\phi_{2}(t)-\phi_{3}(t)=\Phi_{23}$ (very similar curves are obtained for the other differences) calculated with 500 Monte Carlo iterations and for $\pm 2 \%$ coupling variability, i.e. $a=0.02$ : the resulting phase difference tends to be normally distributed around its mean value 0.625 rad with standard deviation $<$ $1 \%$. Thus, the assumed variability of coupling coefficients does not significantly affect the array phase response. We also verify that the associated phase-noise spectra remain very close to the curve (iii) in Fig. 13 calculated in the absence of variability. It is worth underlining that the whole Monte Carlo simulation with the phase-domain model is accomplished in only 25 minutes while it would require more than one week if it were performed with detailed SpectreRF simulations.

The second issue is related to the importance of tightly matching the frequency $\omega_{1}$ of the locked low-noise oscillator $\mathrm{O}_{1}$ to the free-running frequency $\omega_{0}=2 \pi f_{0}$ of the oscillators in the array. To investigate this aspect, we assume $\omega_{1} \neq \omega_{k}=\omega_{0}$ for $k=2, \ldots, N+1$, and we study the effect of a small frequency detuning $\omega_{0}-\omega_{1}=\Delta \omega=2 \pi \times(1 \mathrm{MHz})$. In the presence of frequency detuning, synchronization of each oscillator in the array with the low-noise one requires that, for $t \rightarrow \infty$, the following condition holds [13]

$$
\omega_{1} t+\omega_{1} \alpha_{1}(t)-\omega_{k} t+\omega_{k} \alpha_{k}(t)=\Phi_{1 k},
$$

with $\Phi_{1 k}$ being a constant. This means that, at synchonization, the phase differences

$$
\phi_{1}(t)-\phi_{k}(t)=\omega_{k} t-\omega_{1} t+\Phi_{1 k}=\Delta \omega t+\Phi_{1 k}
$$

for $k=2, \ldots, N+1$ should contain a term $\Delta \omega t$ growing linearly with time that compensates for frequency detuning. For oscillators within the array, instead, mutual synchronization condition remains as in (7) for $k, j \in(2, \ldots, N+1)$.

Fig. 15 shows the phase response of the injected array in the presence of the assumed frequency detuning and for the coupling parameters $g=10^{-5} \Omega^{-1}$ and $g_{r}=10^{-6} \Omega^{-1}$ considered so far. With these parameters, condition (30) is not met and the oscillators within the arrays do not synchronize with $\mathrm{O}_{1}$. As a result, the phase differences among nearby oscillators, do not reach constant values but exhibit fluctuations $( \pm 10 \%$ ) with period $2 \pi / \Delta \omega$, as shown in Fig. 16 .

Array synchronization with $\mathrm{O}_{1}$ is completely recovered if coupling coefficients values are increased to $g=2.5 \cdot 10^{-5} \Omega^{-1}$ and $g_{r}=2.5 \cdot 10^{-6} \Omega^{-1}$. With these parameters, the array phase response shown in Fig. 17 satisfies synchronization condition (30) and phase differences among nearby oscillators reach the prescribed constant phase separations. We also verified that, in this condition, the resulting phase-noise spectra are still very similar to the curve (iii) shown in Fig. 13.

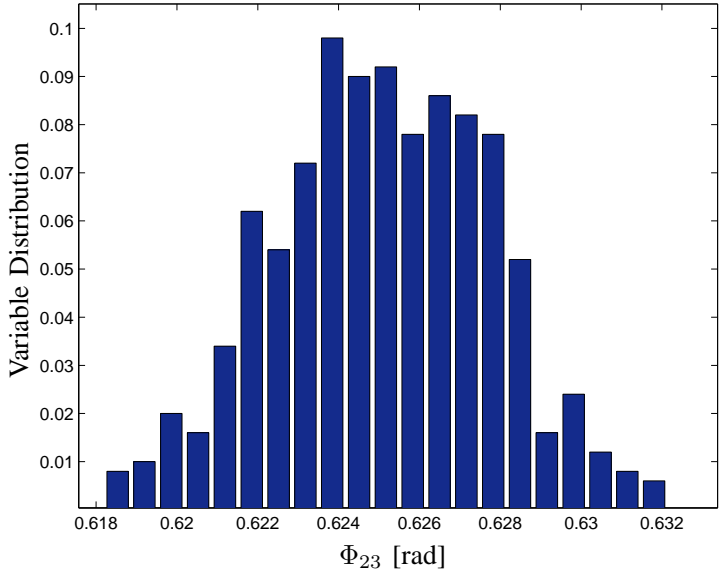

Fig. 14. Statistical distribution of the phase difference $\Phi_{23}=\phi_{2}(t)-\phi_{3}(t)$ due to coupling coefficients variability.

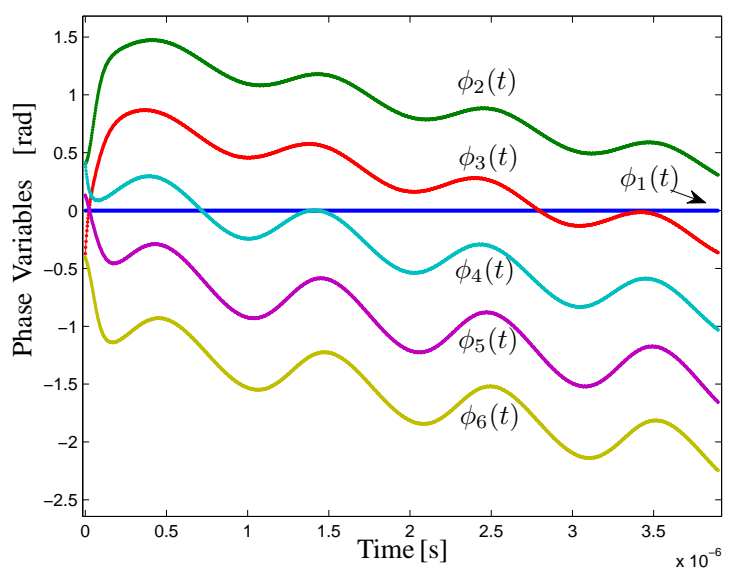

Fig. 15. Phase response of the array in the presence of frequency detuning $\Delta \omega$ and for coupling $g=10^{-5} \Omega^{-1}$ and $g_{r}=10^{-6} \Omega^{-1}$.

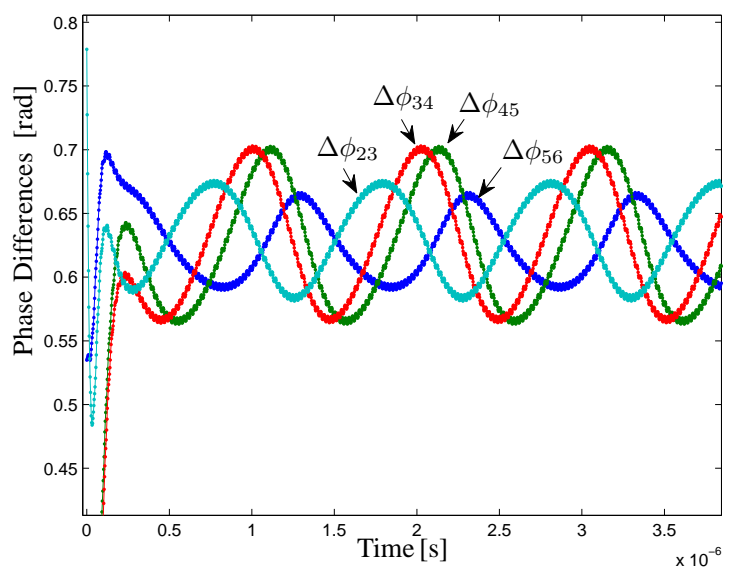

Fig. 16. Phase differences $\Delta \phi_{k, k+1}(t)=\phi_{k}(t)-\phi_{k+1}(t)$ among nearby oscillators in the array when they do not synchronize with $\mathrm{O}_{1}$. 


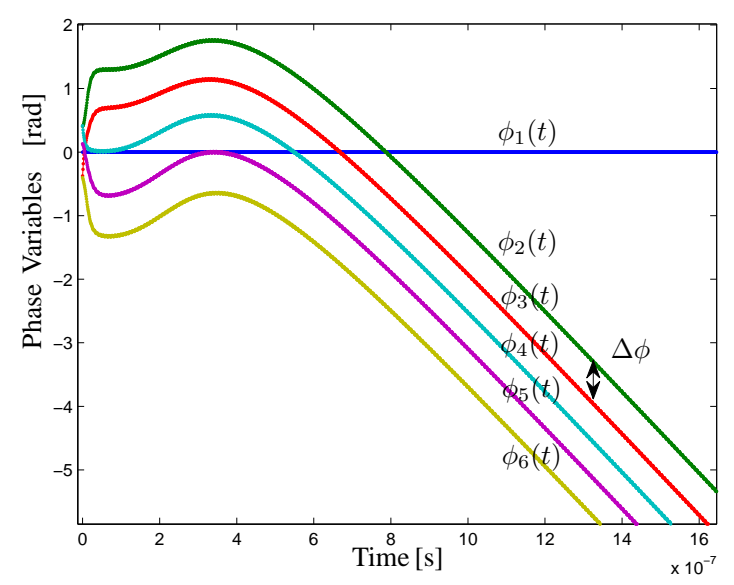

Fig. 17. Phase response of the array in the presence of frequency detuning $\Delta \omega$ and for coupling $g=2.5 \cdot 10^{-5} \Omega^{-1}, g_{r}=2.5 \cdot 10^{-6} \Omega^{-1}$.

\section{CONCLUSION}

Phase-noise generation mechanism in arrays of multi-phase LC oscillators has been studied using the concept of noise transfer function. We have shown how such functions admit fraction expansions with poles that are the eingevalues of a matrix A only dependent on the coupling coefficients and achieved steady-state phase separations. The proposed analysis allows handling the case of chain arrays enhanced by the injection of a clean low-noise signal. By exploiting phase-domain simulations and noise transfer functions we have shown how a properly dimensioned chain array with external injection can provide the prescribed phase separation while significantly improving the output phase noise of the oscillators in the chain. Some results have been presented for LC oscillators in CMOS technology, and they can be applied to the wider family of resonant oscillators, which includes resonant nano-oscillators fabricated in emerging technologies such as MEMS resonant body transistor [23], [24].

\section{APPENDIX}

Starting from (15), we sketch here the steps leading to phase noise expressions (17), (19) and (20). Interested readers can find more details in [6]. To this aim, we substitute (16) into (15) and, exploiting the fact that $\tau_{k}(t)<<\widetilde{\alpha}_{k}(t)$, we linearize equations using Taylor expansion truncated to first order terms

$\Gamma_{k}\left(t+\widetilde{\alpha}_{k}(t)+\tau_{k}(t)\right) \approx \Gamma_{k}\left(t+\widetilde{\alpha}_{k}(t)\right)+\dot{\Gamma}_{k}\left(t+\widetilde{\alpha}_{k}(t)\right) \tau_{k}(t)$ $V_{k}\left(t+\widetilde{\alpha}_{k}(t)+\tau_{k}(t)\right) \approx V_{k}\left(t+\widetilde{\alpha}_{k}(t)\right)+\dot{V}_{k}\left(t+\widetilde{\alpha}_{k}(t)\right) \tau_{k}(t)$

This results into

$$
\begin{aligned}
& \dot{\tau}_{k}(t)=\left(\dot{\Gamma}_{k}\left(t+\widetilde{\alpha}_{k}(t)\right) \cdot \sum_{j=1}^{N} g_{k j} V_{j}\left(t+\widetilde{\alpha}_{j}(t)\right)\right) \tau_{k}(t) \\
& +\Gamma_{k}\left(t+\alpha_{k}(t)\right) \cdot \sum_{j=1}^{N} g_{k j} \dot{V}_{j}\left(t+\widetilde{\alpha}_{j}(t)\right) \tau_{j}(t)+n_{k}(t)
\end{aligned}
$$

In view of (3) and (5), that are valid for harmonic oscillators, (32) is transformed into

$$
\begin{aligned}
\dot{\tau}_{k}(t)= & \left\{\Gamma_{M} V_{M} \omega_{0} \cos \left[\omega_{0}\left(t+\widetilde{\alpha}_{k}\right)\right]\right. \\
& \left.\cdot \sum_{j=1}^{N} g_{k j} \cos \left[\omega_{0}\left(t+\widetilde{\alpha}_{j}\right)\right]\right\} \tau_{k}(t) \\
& +\Gamma_{M} V_{M} \omega_{0} \cos \left[\omega_{0}\left(t+\widetilde{\alpha}_{k}-\pi / 2\right)\right] \\
& \cdot \sum_{j=1}^{N} g_{k j} \cos \left[\omega_{0}\left(t+\widetilde{\alpha}_{j}\right)+\pi / 2\right] \tau_{j}(t)+n_{k}(t) .
\end{aligned}
$$

We then use averaging [21], [22] and keep only the lowfrequency terms arising from the cosine products in (33), obtaining

$$
\begin{aligned}
\dot{\tau}_{k}(t) \approx & B\left(\sum_{j=1}^{N} g_{k j} \cos \left(\Phi_{k j}\right)\right) \cdot \tau_{k}(t) \\
& -B \sum_{j=1}^{N} g_{k j} \cos \left(\Phi_{k j}\right) \cdot \tau_{j}(t)+n_{e q}^{k}(t) .
\end{aligned}
$$

where $B=\omega_{0} \Gamma_{M} V_{M} / 2$ and where $\Phi_{k j}$ are defined in (7).

Denoting $\vec{\theta}(t)$ the vector that collects all excess phase variables $\theta_{k}(t)=\omega_{0} \tau_{k}(t)$ and $\vec{n}(t)$ the vector of macro noise sources, from (34) we deduce

$$
\frac{d}{d t} \vec{\theta}(t)=\mathbf{A} \cdot \vec{\theta}(t)+\omega_{0} \vec{n}(t)
$$

where the elements of matrix $\mathbf{A} \in \mathbb{R}^{N \times N}$ are given by

$$
\begin{aligned}
& a_{k k}=B \sum_{j=1, j \neq k}^{N} g_{k j} \cos \left(\Phi_{k j}\right) \\
& a_{k j}=-B g_{k j} \cos \left(\Phi_{k j}\right) \quad \text { for } k \neq j .
\end{aligned}
$$

Finally, Fourier transforming (35) and passing to power noise, the closed-form expression (17) is obtained with matrix $\mathbf{T}(f)$ defined as in (19).

We also observe how entries of matrix $\mathbf{A}$ are decided by the array topology, which is described by $g_{k j}$ coupling coefficients, and by the phase-difference values $\Phi_{k j}$ (7) that are reached at synchronization. Matrix $\mathbf{A}$ is singular with rank $N-1$ and thus has a null eigenvalue, let's say $\lambda_{1}=0$. In addition, from (35) we see that the eigenvalues of $\mathbf{A}$ govern the dynamics induced by any perturbation of the steady-state solution of (6) [20]. As a result, for any phase separation $\Phi_{k j}$ that corresponds to a stable solution of (6), i.e. that can be obtained by numerically simulating (6) in time, the eigenvalues $\lambda_{k}$ for $k=2, \ldots, N$ should necessarily have negative real part, i.e. $\Re\left(\lambda_{k}\right)<0$.

\section{REFERENCES}

[1] R. van de Beek, E. Klumperink, C. Vaucher, and B. Nauta, "Low-jitter clock multiplication: a comparison between PLLs and DLLs," IEEE Trans. Circuits Syst. II, Analog Digit. Signal Process., vol. 49, no. 8, pp. 555-566, Aug. 2002. 
[2] K. Lee et al., "A single-chip 2.4-GHz direct-conversion CMOS receiver for wireless local loop using multiphase reduced frequency conversion technique", IEEE J. Solid-State Circuits, vol. 36, no. 5, pp. 800-809, May 2001.

[3] L. Romanò, S. Levantino, C. Samori, A. L. Lacaita, "Multiphase LC Oscillators," IEEE Trans. Circuits and Syst. I: Regular Papers, vol. 53, no. 7, pp. 1579-1588, Jul 2006.

[4] P. Maffezzoni, B. Bahr, Z. Zhang, and L. Daniel, "Oscillator Array Models for Associative Memory and Pattern Recognition," IEEE Trans. Circuits and Syst. I: Regular Papers, vol. 62, no. 6, pp. 1591-1598, Jun. 2015.

[5] H. C. Chang, X. Cao, U. K. Mishra, R. York, "Phase Noise in Coupled Oscillators: Theory and Experiment," IEEE Tran. Microwawe Theory and Techniques, vol. 45, no. 5, pp. 605-615, May 1997.

[6] P. Maffezzoni, B. Bahr, Z. Zhang, and L. Daniel, "Analysis and Design of Weakly Coupled Oscillator Arrays Based on Phase-Domain Macromodels," IEEE Trans. Computer-Aided-Design of Integrated Circuits and Systems, vol. 34, no. 1, pp. 77-85, Jan. 2015.

[7] F. X. Kaertner, "Analysis of White and $f^{-\alpha}$ Noise in Oscillators," International Journal of Circuit Theory and Applications, vol. 18, pp. 485-519, 1990.

[8] A. Demir, A. Mehrotra and J. Roychowdhury, "Phase Noise in Oscillators: A Unifying Theory and Numerical Methods for Characterisation," IEEE Trans. Circuits and Syst. I, vol. 47, no. 5, pp. 655-674, May 2000.

[9] M. Bonnin, F. Corinto, M. Gilli, "Phase Space Decomposition for Phase Noise and Synchronization Analysis of Planar Nonlinear Oscillators," IEEE Trans. Circuits and Syst. II: Express Briefs, vol. 59, no. 10, pp. 638-642, Oct. 2012

[10] M. Bonnin, F. Corinto, "Phase Noise and Noise Induced Frequency Shift in Stochastic Nonlinear Oscillators" IEEE Trans. Circuits and Syst. I: Regular Papers, vol. 60, no. 8, pp. 2104 - 2115, Aug. 2013.

[11] M. M. Gourary, S. G. Rusakov, S. L. Ulyanov, et. al. "Smoothed Form of Nonlinear Phase Macromodel for Oscillators," IEEE/ACM Int. Conf. on Computer-Aided Design, San Jose, CA, pp. 807-814, 2008.

[12] D. Harutyunyan, J. Rommes, J. ter Maten, W. Schilders, "Simulation of Mutually Coupled Oscillators Using Nonlinear Phase Macromodels Applications," IEEE Trans. on Computer-Aided-Design of Integrated Circuits and Systems, vol. 28, no. 10, pp. 1456-1466, Oct. 2009.

[13] A. Pikovsky, M. Rosenblum, J. Kurths, Synchronization, Cambridge Univ. Press, UK 2001

[14] P. Maffezzoni, S. Levantino, "Phase Noise of Pulse Injection-Locked Oscillators," IEEE Trans. Circuits and Syst. I: Regular Papers, vol. 61, no. 10, pp. 2912-2919, Oct. 2014.

[15] G. B. Ermentrout, N. Kopell, "Frequency plateaus in a chain of weakly coupled oscillators," SIAM J. Math. Anal., vol 15, no. 2, pp. 215-237, 1984.

[16] S. Levantino, P. Maffezzoni, "Computing the Perturbation-ProjectionVector of Oscillators via Frequency Domain Analysis," IEEE Trans. on Computer-Aided-Design of Integrated Circuits and Systems, vol. 31, no. 10, pp. 1499-1507, Oct. 2012

[17] A. Demir, "Computing Timing Jitter From Phase Noise Spectra for Oscillators and Phase-Locked Loops With White and $1 / f$ Noise IEEE Trans. Circuits and Syst. I, vol. 53, no. 9, pp.1859-1874, Sep. 2006.

[18] P. Maffezzoni, S. Levantino, "Analysis of VCO Phase Noise in ChargePump Phase-Locked Loops," IEEE Trans. Circuits and Syst. I: Regular Papers, vol. 59, no. 10, pp. 2165-2175, Oct. 2012.

[19] Virtuoso Spectre Circuir Simulator RF Analysis User Guide, Product Version 7.2, Cadence Design Syst., San Jose, CA, May 2010.

[20] M. Farkas, Periodic Motions, Springer-Verlag, 1994.

[21] M. I. Freidlin and A. D. Wentzell, Random Perturbations of Dynamical Systems. Berlin, Germany: Springer-Verlag, 1984.

[22] P. Vanassche, G. Gielen, W. Sansen, "On the difference between two widely publicized methods for analyzing oscillator phase behavior," Proc. ICCAD 2002, Nov. 2002, pp. 229-233.

[23] R. Marathe, B. Bahr, W. Wang, Z. Mahmood, L. Daniel, and D. Weinstein, "Resonant Body Transistors in IBMs $32 \mathrm{~nm}$ SOI CMOS Technology," Journal of Microelectromechanical Systems, vol. 23, no. 3, pp. 636-650, June 2014.

[24] B. Bahr, R. Marathe, D. Weinstein, "Theory and Design of Phononic Crystals for Unreleased CMOS-MEMS Resonant Body Transistors," Journal of Microelectromechanical Systems, vol. 24, no. 5, pp. 15201532, Oct. 2015.

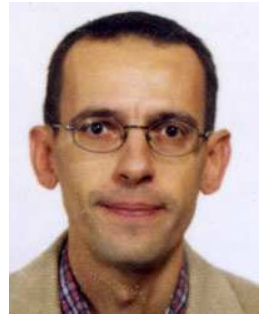

Paolo Maffezzoni (M'08-SM'15) received the Laurea degree (summa cum laude) in Electrical Engineering from Politecnico di Milano, Italy, in 1991 and the Ph.D. degree in Electronic Instrumentation from Universita' di Brescia, Italy, in 1996. Since 1998, he has been with Politecnico di Milano where he is an Associate Professor of Electrical Engineering. His research interests include analysis, simulation and design of nonlinear dynamical circuits and systems, oscillating devices, synchronization, stochastic simulation. He has over 120 research publications among which 64 papers in international journals. He has served as an Associate Editor for the IEEE Transactions on Computer-Aided Design of Integrated Circuits and Systems.

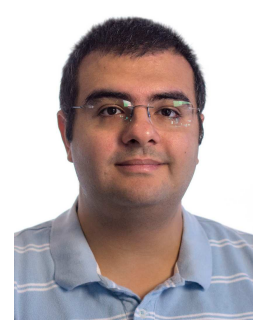

Bichoy Bahr (S'10) received the B.Sc. degree with honors in 2008 and the M.Sc. degree in 2012, both in electrical engineering, from Ain Shams University, Cairo, Egypt. He worked as an Analog/Mixed Signal and MEMS Modeling/Design Engineer at MEMS Vision, Egypt. Mr. Bahr is currently working towards the Ph.D. degree in the Department of Electrical Engineering at Massachusetts Institute of Technology (MIT), Cambridge, MA, USA. He is a research assistant in the HybridMEMS group, MIT. Mr. Bahr's research interests include the design, fabrication, modeling and optimization of monolithically integrated unreleased MEMS resonators, in standard ICs technology. He is also interested in multi-GHz MEMS-based monolithic oscillators, coupled oscillator-arrays and unconventional signal processing.

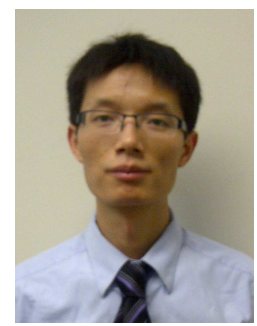

Zheng Zhang (S'09-M'15) received his Ph.D degree (2015) in Electrical Engineering and Computer Science from Massachusetts Institute of Technology (MIT), Cambridge, MA, his M.Phil degree (2010) in Electrical and Electronic Engineering from the University of Hong Kong, Hong Kong, and his B. Eng degree (2008) in Electronics from Huazhong University of Science and Technology, Wuhan, China. He is currently a postdoc associate with the Mathematics and Computer Science Division at Argonne National Laboratory, Argonne, IL. His research interests include high-dimensional uncertainty quantification, tensors and data analysis. Applications of interest include nano-scale devices, circuits and systems, energy systems and biomedical computation. Dr. Zhang received the 2015 Doctoral Dissertation Seminar Award (i.e., best dissertation award) from the Microsystems Technology Laboratory of MIT, the 2014 Best Paper Award from IEEE Transactions on CAD of Integrated Circuits and Systems, the $2011 \mathrm{Li} \mathrm{Ka}$ Shing Prize (university best M.Phil/Ph.D thesis award) from the University of Hong Kong, the 2010 Mathworks Fellowship from MIT, and three additional best paper nominations in international conferences. His industrial research experiences include Coventor Inc. and Maxim-IC.

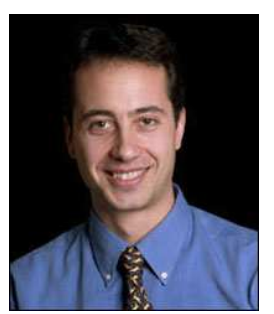

Luca Daniel (S'98-M'03) received the Ph.D. degree in Electrical Engineering from the University of California, Berkeley, in 2003. He is currently a Full Professor in the Electrical Engineering and Computer Science Department of the Massachusetts Institute of Technology (MIT). Industry experiences include HP Research Labs, Palo Alto (1998) and Cadence Berkeley Labs (2001). His current research interests include integral equation solvers, uncertainty quantification and parameterized model order reduction, applied to RF circuits, silicon photonics,

MEMs, Magnetic Resonance Imaging scanners, and the human cardiovascular system. Prof. Daniel was the recipient of the 1999 IEEE Trans. on Power Electronics best paper award; the 2003 best $\mathrm{PhD}$ thesis awards from the Electrical Engineering and the Applied Math departments at UC Berkeley; the 2003 ACM Outstanding Ph.D. Dissertation Award in Electronic Design Automation; the 2009 IBM Corporation Faculty Award; the 2010 IEEE Early Career Award in Electronic Design Automation; the 2014 IEEE Trans. On Computer Aided Design best paper award; and seven best paper awards in conferences. 